\title{
A haptic digital tool to assist the design, planning and manufacture of micro- and nano-structures
}

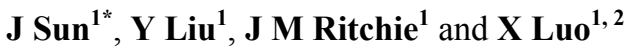 \\ ${ }^{1}$ Institute of Mechanical, Process and Energy Engineering, School of Engineering and Physical Sciences, \\ Heriot-Watt University, Edinburgh, EH14 4AS, UK. \\ ${ }^{2}$ Department of Design, Manufacture and Engineering Management, University of Strathclyde, Glasgow \\ G1 1XQ, UK.
}

\begin{abstract}
This work addresses the engineering demands for precise interactive process planning for the manufacture of high precision in the manufacturing of 3D micro- and nano-products. It outlines the development of a digital tool based on a haptics-based human machine interface. This interface is underpinned using an empirical model derived from a series of experimental results obtained from a focused ion beam (FIB) machine through a series of experiments which produced the surface topographies of a number of micro- and nano-grating arrays; these were systemically analysed to provide an underpinning empirical database which could be accessed via the haptic process planning system. On accessing these data, highly accurate geometrical models are then input into a haptics human machine interface to emulate the FIB machining process as defined by the customized fabrication parameters. The haptic planning system predicts and simulates the shape and form of the finished product profiles as the haptic device is passed across a virtual substrate. Once the simulation is complete and the outputs verified by the user, the system then automatically outputs a stream file to drive the FIB process to produce the surface topographies required. The simulation results reveal that compared with trial and error method, the haptics-based simulation method is a reliable way to plan and optimize the fabrication parameters in design and manufacture of micro- and nano-gratings by FIB machining cost-effectively. This could potentially provide substantial lead time and cost reductions associated with these currently inherently expensive unit cost products as well as demonstrate a novel tool not only for the planning of nano-machining cutting sequences but also for the interactive design of such products. This work also highlights the benefits of an empirical model as a means of underpinning and supporting real-time haptics interaction, something not possible using more numerical-based techniques.
\end{abstract}

Key words: design and manufacture, digital tool, focused ion beam, haptics, process planning.

* Corresponding author: Institute of Mechanical, Process and Energy Engineering, School of Engineering and Physical Sciences, Heriot-Watt University, Edinburgh, EH14 4AS, UK.

email: $\underline{\text { Jining.Sun@,hw.ac.uk }}$ 


\section{INTRODUCTION}

Focused ion beam (FIB) machining is an enabling technology for the fabrication of 3D microand nano-products. It can also be used to correct any form errors left by previous rougher, less accurate fabrication processes. The spot size of the FIB can be as small as $5 \mathrm{~nm}$ and ion polishing is especially suitable for the final "figuring" of optics where extremely tight form accuracy is required. However, the control of FIB machining for 3D products is very sophisticated. The process suffers from, i.e. the redeposition effect, the angular dependence of sputter yield and the Gaussian beam profile, which significantly limit machining accuracy. The "trial and error method" " "divergence compensation method" 2 and "depth control method" 3 are generally used in FIB machining, particularly when fabricating structures with complex geometries. However, these geometries are rarely used in industrial applications. Basic micro- and nano-structures such as dots, lines and regular rectangle cavities are much more widely used in photonics, micro- and nano-machining applications, the integrated circuit (IC) industry and material sciences. For example, in IC editing, the line width cut by a FIB needs to be extremely precise to avoid overlapping with the adjacent circuit. It is necessary to develop reliable and cost-effective methods to design and optimize the fabrication parameters in these currently inherently expensive unit cost products.

Computer aided process planning is the step between computer aided design and the physical output through fabrication. Traditional modelling utilizes mathematical models in an attempt to predict certain behaviours within a system ${ }^{4}$. While there are many computer simulations available, a common trait that is present throughout is that they produce representative scenarios and behaviours of a model ${ }^{5}$. These simulations have a crucial role to play in the process of product and manufacturing process design, supporting the reduction of overall costs through less prototyping ${ }^{6}$. However, they have one fundamental drawback if they are to be used in real time interaction and simulation: they require substantial amounts of processing time and iterations where geometry modifications and visual changes are required instantly.

Haptics integrated into computer simulations for design and process planning can provide the user with a more complete experience because of its tactile feedback, whereas most commercial modelling simulations focus mainly on the visual aspects ${ }^{7}$. Therefore, these computer simulations and virtual environments are now being modified to include haptic devices in order to provide a sense of feel for a more natural and insightful understanding of both products and processes ${ }^{8}$. By fitting the experimental FIB machining data into a database model and embedding this within a haptics simulation program, the user is able to simulate the results of the FIB machining process in real time without the need for multiple iterations followed by analysis to determine the results. This will provide the user with a semi-automated tool for planning to rapidly determine the resulting structures, shapes and corresponding tool path while automatically generating a plan and machine control data.

In this work, the relationship between the fabrication parameters and the machined surface topography is systematically studied through a FIB machining experiment. Based on the experimental results, a database was created for the planning of the manufacturing process through a digital tool. This tool will be researched to evaluate their effectiveness in enhancing the 
quality, throughput, controllability and productivity of FIB machining. One intuitive technology that can supplement such system is haptics which includes both hardware and software to enable manual interactions with a product or process embedded within a $3 \mathrm{D}$ virtual world ${ }^{9}$. Haptics has been researched in a range of processes in manufacturing such as machining ${ }^{10}$ and assembly ${ }^{11}$. The intuitive nature of the haptics also makes it a good choice for automated processes which require front-end human input for planning purpose, such as tool path planning in FIB machining, because it enhances the experience of the user at the human scale through allowing them to focus on path planning whilst encountering and experiencing the physics processes at the micro- or nano-scale.

\section{DESIGN OF EXPERIMENTS}

This research work focused on using an experimental method to analyse the surface topography evolution under different fabrication parameters in the FIB machining process and then using these data as a foundation for a haptic FIB planning system. The profiles of the structures were described by certain characteristic parameters determined using numerical simulation methods. The experimental results were then input into a database which could be accessed by a hapticsbased simulation program. The overall approach to this study is illustrated in Figure 1.

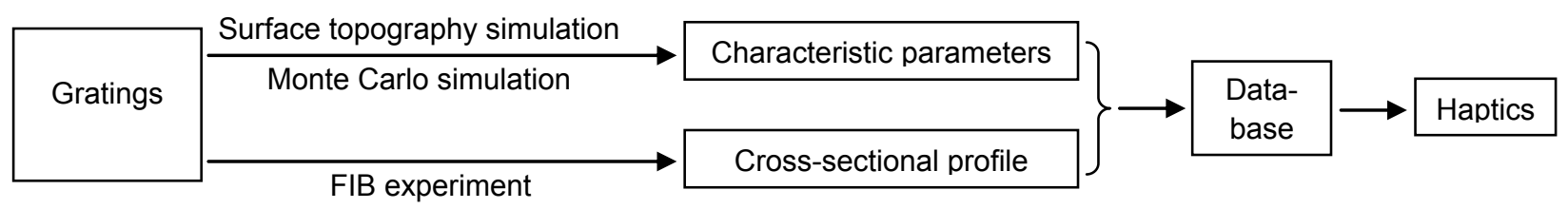

Figure 1 Block diagram of the proposed planning process.

\subsection{Determination of the characteristic parameters}

Micro- and nano-gratings can be described by a set of characteristic parameters. In this work, the surface topography evolution under ion beam bombardment was studied using a numerical simulation method. This method was based on a physical sputtering model, where a beam of ions was incident on the target surface. The total amount of sputtered atoms per incident ion, which primarily depends on the ion incident angle, can be calculated through a Monte Carlo simulation. Some of these sputtered atoms may adhere to the machined surface, resulting in the well-known redeposition effect. Therefore, the surface generation in FIB machining is a combination of the material removal and redeposition processes. The physical sputtering process is illustrated in Figure 2. This model can be further combined with a surface topography simulation method to accurately track the surface evolution under certain fabrication parameters. Experimental evaluation results have revealed that the model and simulation program developed can precisely describe the generation of a 3D surface in FIB machining process (with less than $7 \%$ simulation 
error) ${ }^{12}$. According to these, it is known that gratings can be characterized by three independent parameters: the mouth width, depth and bottom width.

- The mouth width is the diameter of the nanodot on the machined surface. The simulation and experimental results both revealed that it is much larger than the beam spot size due to the Gaussian distribution of the incident ion beam. The widely extended section in the beam, which is called the "beam tail" or the "beam skirt", contributes significantly to widen the mouth width during the machining process.

- The depth of the nanodot is defined as the maximum distance between the substrate surface and the bottom of the nanodot. In nanometre scale, it increases linearly with the incident ion dose when taking the redeposition effect into consideration.

- The bottom width is defined as the length of the flat part at the bottom of the nanodot with the bottom width and the beam diameter positively correlated.

However, since the simulation process is very time consuming, the dimensions of these structures calculated by the simulation program are at the nano-scale and it is still a challenge to evaluate the surface topography evolution at the micro-scale. This work aimed to schematically build a database of the profiles of micro- and nano-structures fabricated by FIB machining through an alternative empirical and experimental method. The database could then be accessed by appropriate digital tool, based on a haptics-human machine interface, for the real-time evaluation of the surface topography. As the FIB machining process follows the same principles at both micro- and nano-scales, the same characteristic parameters can be applied to the experimentation parameters subsequently used in the database to describe the structure profiles.

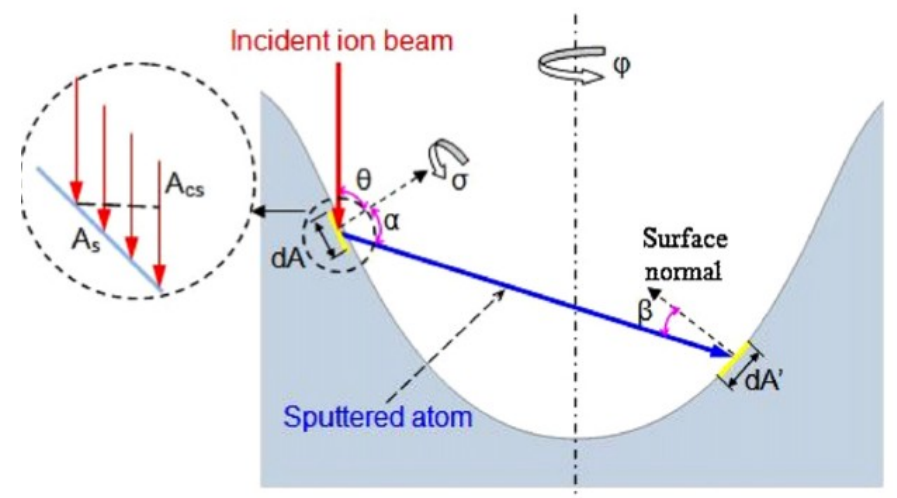

Figure 2 Schematic illustration of the generation mechanism of a machined surface fabricated by FIB ${ }^{12}$.

\subsection{Experimental Methodology and Database Creation}

The experiment was carried out on a FEI Quanta 3D FEG dual beam system FIB machine. Before the experiment, all of the adjustable parameters such as the focusing lens, focus point, aperture and stigmation were aligned properly. Micro- and nano-grating arrays with depth ranging from $150 \mathrm{~nm}$ to $7 \mu \mathrm{m}$ were fabricated on a silicon substrate. In the machining process, fabrication parameters including ion current, dwell time, pixel spacing and scanning passes were all changed 
schematically. For each combination of fabrication parameters, the cross-sectional profile of the machined structure was measured using the same procedure:

1. A layer of platinum was deposited onto the local structure by ion beam assisted chemical vapour deposition. The platinum deposition was helpful in protecting the structures during the cross sectioning process. In addition, the contrast of the SEM micro image was also enhanced due to the existence of the platinum layer, which made it much easier to determine the edge of the profiles.

2. Cross sections were cut across the target structures by FIB machining. They were cut in a stair step fashion to allow the exposed layers to be seen with the electron beam when the sample is tilted.

3. A much lower ion current $(100 \mathrm{pA})$ was applied to further polish the cross sections for a final cut.

4. Cross-sectional profiles of the structures were then measured by SEM at a $52^{\circ}$ tilted angle relative to the substrate surface. For each structure, the mouth width, the depth and the bottom width were measured by the field emission SEM equipped with the system.

The entire experimental process is demonstrated in Figure 3.

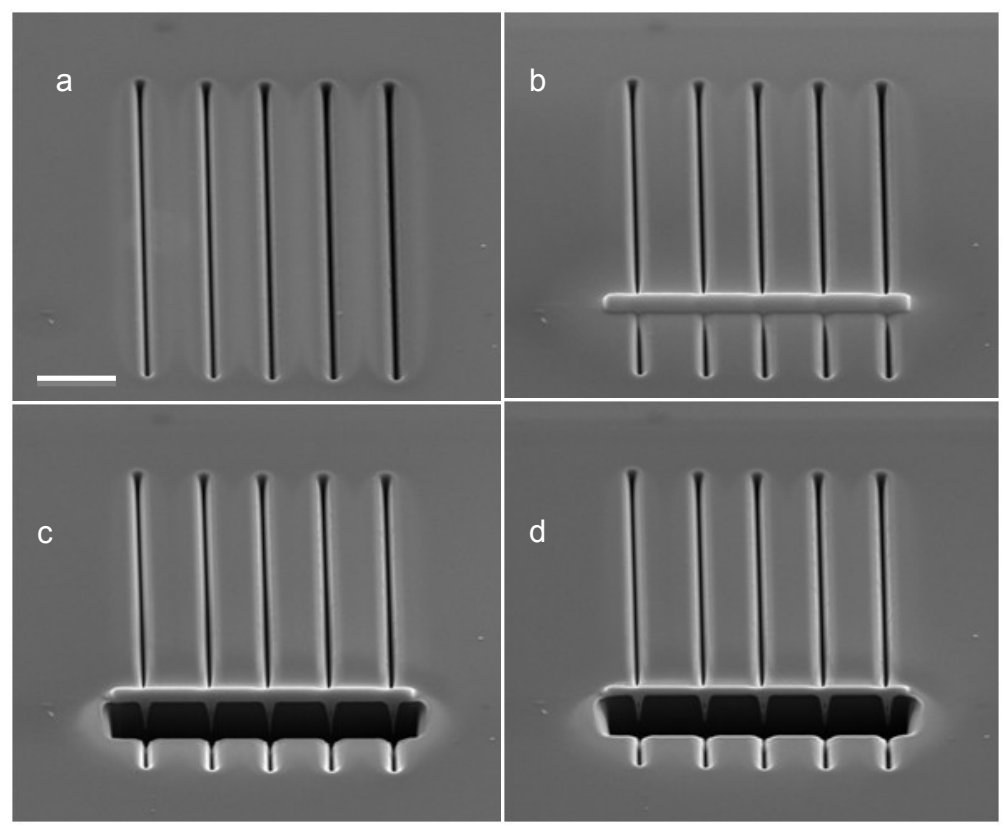

Figure 3 Experimental processes for the measurement of cross-sectional profile of the machined structures.

(a) nano-grating array fabricated by FIB machining; (b) platinum deposition process; (c) cross section milled across the nano-grating array; (d) ion beam polishing process for the final measurement. (the scale bar in Figure $3 \mathrm{a}$ is $1 \mu \mathrm{m}$ )

For this experiment the ion beam energy was fixed at $30 \mathrm{keV}$, which is the most widely used energy in the IC industry. Other fabrication parameters such as ion current, dwell time, scanning pitch and number of scanning passes were all investigated in this work. To systematically study the interactions of the fabrication parameters, ion currents of $0.1 \mathrm{nA}, 1 \mathrm{nA}$ and $5 \mathrm{nA}$ were chosen as representatives of low-current, mid-current and high-current. For each ion current, the dwell time 
was varied from $1 \mu \mathrm{s}$ to $50 \mu \mathrm{s}$, while the scanning pitch was fixed at the beam diameter $(0 \%$ overlap) and beam radius (50\% overlap), respectively. The number of scanning passes was fixed at 10,000 .

Since the intensity of the ion beam follows a Gaussian distribution, the cross-sectional profile of the structures with low aspect ratio also follows the same type of distribution. This phenomenon has been used to measure the ion beam profile by many former researchers ${ }^{13}$. As the aspect ratio increases, the ion incident angle increases from $0^{\circ}$ to almost $90^{\circ}$ which leads to an intense variation on the local sputter yield. Therefore, the cross-sectional profile of the structures starts to diverge from the original Gaussian distribution. This effect can be corrected by introducing a correction factor $k(x)$ to the Gaussian distribution:

$$
f(x)=\frac{k(x)}{\sigma \sqrt{2 \pi}} e^{-\frac{1}{2}\left(\frac{x}{\sigma}\right)^{2}}
$$

where $\sigma^{2}$ is the variance of the Gaussian distribution; $x$ represents the lateral coordinate along the cross section; $f(x)$ is the cross-sectional profile of the machined structure.

Further investigation revealed that during the machining process, the majority of the sidewalls evolved linearly, which signified that the local sputter yield, or the moving speed of the local surface, is almost identical in each scanning pass. Therefore, the points on the sidewalls were equally diverged from the original Gaussian distribution and the correction factor $k(x)$ can be replaced with a constant parameter which is independent of $x$.

According to these characteristic parameters, the cross-sectional profile of the structure was fitted using the following equation:

$$
\left\{\begin{array}{l}
f(x)=d e^{-\frac{1}{2}\left(\frac{x}{\sigma}\right)^{2}} \\
\sigma=\frac{(m+b)}{4 \sqrt{2 \ln 2}}
\end{array}\right.
$$

where $d$ is the depth of the structure and $m$ and $b$ represent the mouth width and the bottom width respectively

\subsection{Haptics}

The hardware of the haptics device include a Sensable Phantom Omni device, which is ideal for the FIB machining simulations because its movements allow the user to move and feel the full degrees of freedom necessary for FIB machining planning. The software is coded in $\mathrm{C}++$ and is multi-threaded, i.e. graphics, physics, and haptics. A $1 \mathrm{kHz}$ rendering frequency is required by the haptics application for more realistic rendering of operations and forces. The software libraries used include OpenSceneGraph (OSG, ${ }^{14}$ ), Open Dynamics Engine (ODE, ${ }^{15}$ ), osgModeling ${ }^{16}$, and OpenHaptics ${ }^{17}$. The user is able to select from an imported database of FIB machining experimental models with a full range of movement in the $\mathrm{X}, \mathrm{Y}$, and $\mathrm{Z}$ directions. The architecture of the software and two segments of the database table are shown in Figure 4 and Table 1 respectively. 


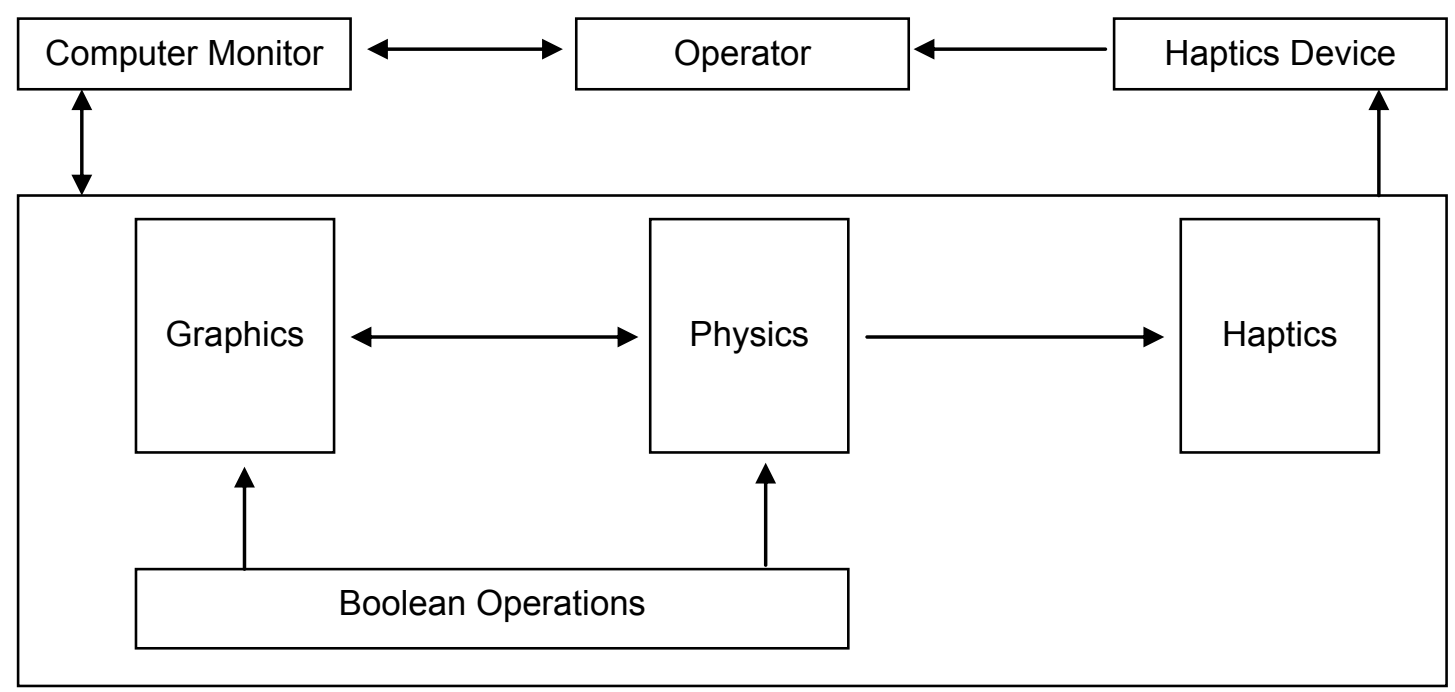

Figure 4 Haptic planning software architecture

Table 1. (a) A segment of the ion current-dose database

\begin{tabular}{|c|c|c|c|c|c|}
\hline $\begin{array}{c}\text { Ion current } \\
(\mathrm{nA})\end{array}$ & $\begin{array}{c}\text { Beam size } \\
(\mathrm{nm})\end{array}$ & $\begin{array}{c}\text { Ion dose } \\
\left(10^{22} \text { ions } / \mu \mathrm{m}^{2}\right)\end{array}$ & $\begin{array}{c}\text { Mouth width } \\
(\mathrm{nm})\end{array}$ & $\begin{array}{c}\text { Bottom width } \\
(\mathrm{nm})\end{array}$ & $\begin{array}{c}\text { Depth } \\
(\mathrm{nm})\end{array}$ \\
\hline 0.1 & 24 & 2.0362 & 280.9 & 59.3 & 230.9 \\
\hline 1 & 44 & 6.0580 & 498.4 & 146.4 & 572.2 \\
\hline 5 & 85 & 8.1165 & 775.3 & 308.5 & 913.5 \\
\hline
\end{tabular}

(b) A segment of the dwell time-dose database

\begin{tabular}{|c|c|c|c|c|}
\hline $\begin{array}{c}\text { Dwell time } \\
(\mu \mathrm{s})\end{array}$ & $\begin{array}{c}\text { Ion Dose } \\
\left(10^{23} \mathrm{ions} / \mu \mathrm{m}^{2}\right)\end{array}$ & $\begin{array}{c}\text { Mouth } \\
(\mathrm{nm})\end{array}$ & $\begin{array}{c}\text { Bottom } \\
(\mathrm{nm})\end{array}$ & $\begin{array}{c}\text { Depth } \\
(\mathrm{nm})\end{array}$ \\
\hline 1 & 0.204 & 282.3 & 67.4 & 169.4 \\
\hline 3 & 0.611 & 353.3 & 54.6 & 466.2 \\
\hline 5 & 1.01 .018 & 400.7 & 58.3 & 626.5 \\
\hline 7 & 1.725 & 408 & 36.4 & 757.7 \\
\hline 10 & 2.036 & 437.1 & 43.7 & 859.6 \\
\hline
\end{tabular}

\section{RESULTS AND DISCUSSION}

Micro- and nano-gratings with various dimensions were successfully fabricated on a silicon substrate as described in the previous section. Figure 5 shows the fabrication results of the microand nano-gratings cut through FIB machining under different ion currents. The cutting depth, mouth width and the bottom width were measured by the field emission SEM equipped within the dual beam system. Figure 5 (a) (c) summarizes the dimensions of the structures fabricated with different dwell time and different scanning pitch ( $0 \%$ overlap and $50 \%$ overlap) under the above three ion currents. The measurements show that both the depth and the mouth width increase with the dwell time from $1 \mu \mathrm{s}$ to $50 \mu \mathrm{s}$, while the bottom width almost remains constant for each ion current. Due to the redeposition effect, the rate of increase of the milling depth slows down, as does the increment of the dwell time. The variations in the scanning pitch also contributed to the depth and the mouth width with identical dwell times; both the depth and the mouth width fabricated under $50 \%$ beam overlap were systematically larger than those fabricated under $0 \%$ 
beam overlap. This is because a higher beam overlap produces a higher concentration of incident ion dose. Considering the beam overlap effect, the total ion dose impact on each milling pixel is actually an accumulation of the ion dose from all of the adjacent milling pixels, as is shown in Figure 6 . The total ion dose $F$ can be calculated using the following equation ${ }^{18}$ :

$$
F=\frac{4 \ln (2) \times t \times s p \times I}{\pi \times e \times F W H M^{2}} \sum_{i, j, p} \exp \left[4 \times \ln (2) \times \frac{\left(x_{i j}-x_{p}\right)^{2}+\left(y_{i j}-y_{p}\right)^{2}}{F W H M^{2}}\right]
$$

where $t$ and $s p$ are the dwell time and the scanning passes respectively; $I$ is the ion current; e represents the charge of an electron; $x_{p}$ and $y_{p}$ are the coordinates of the beam centre position; and FWHM is the beam diameter.

The relationships between the ion dose and the structures' dimensions are shown in Figure 5 (d)(f). For each current, the dimensions of the structures fabricated under different scanning pitch fitted well onto a characteristic curve. This phenomenon leads to a very important conclusion that, for each ion current, the dimensions of the gratings are only related to the ion dose in the same scanning passes. 

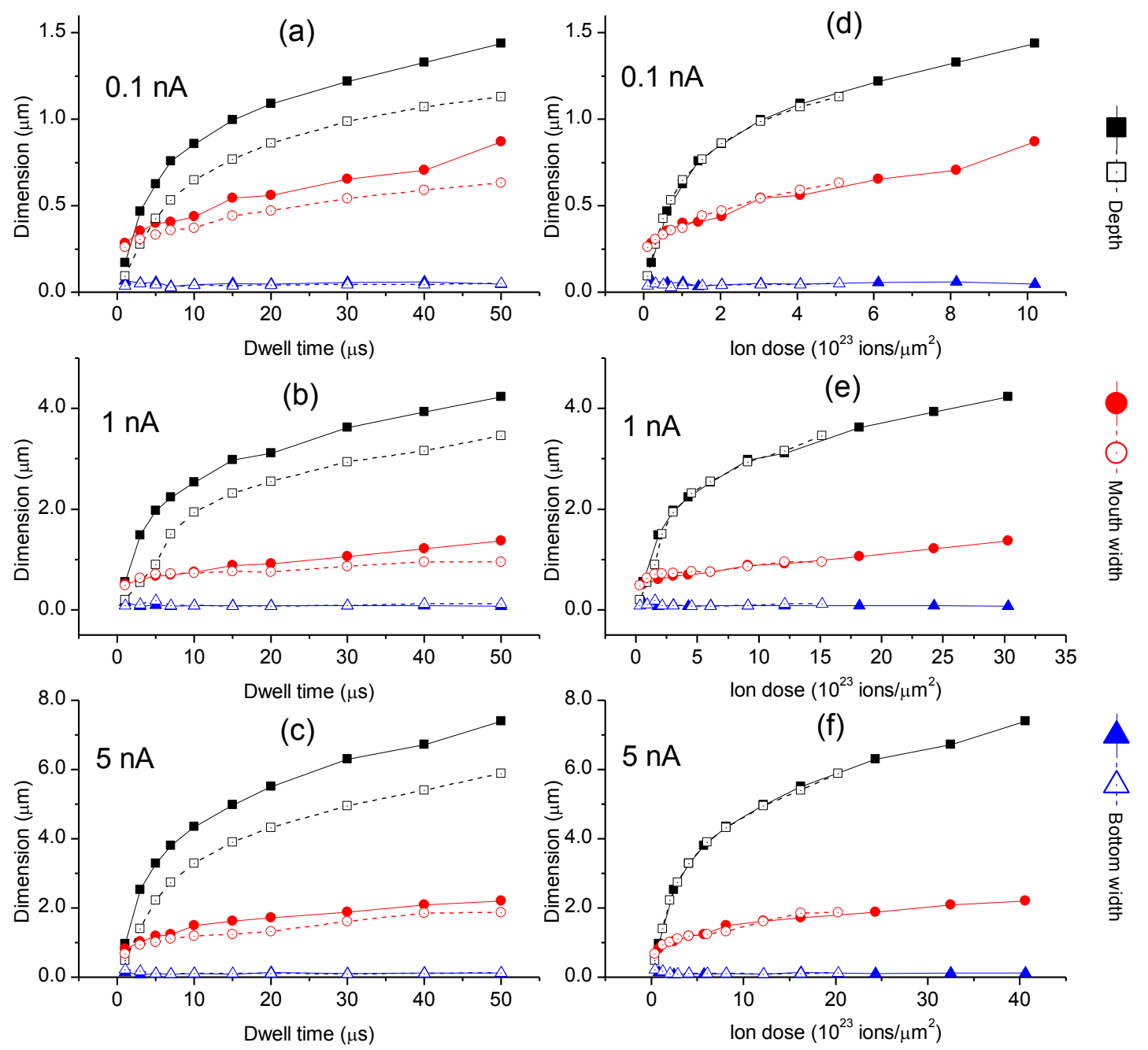

Figure 5 Fabrication results of the micro- and nano-gratings through FIB machining under different ion currents: (a)-(c) show the relationship between dwell time and the dimensions; (d)-(f) show the relationship between ion dose and the dimensions; the gratings fabricated using a $50 \%$ overlap are illustrated by the solid filled points while the white filled points represent a $0 \%$ overlap. 


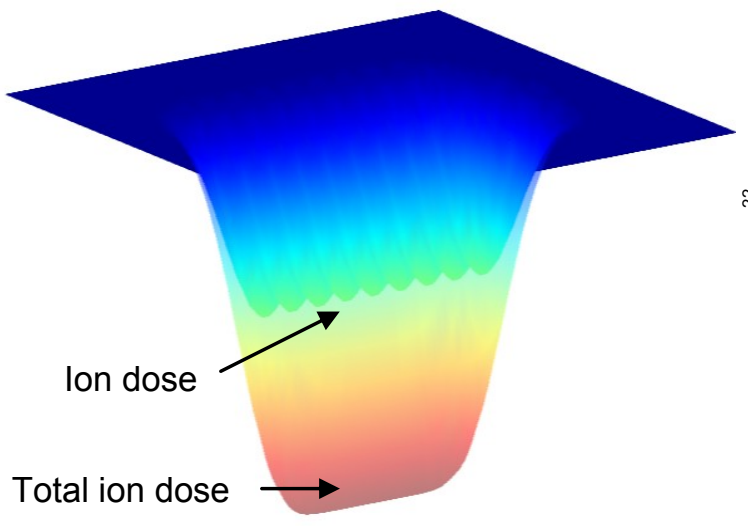

(a)

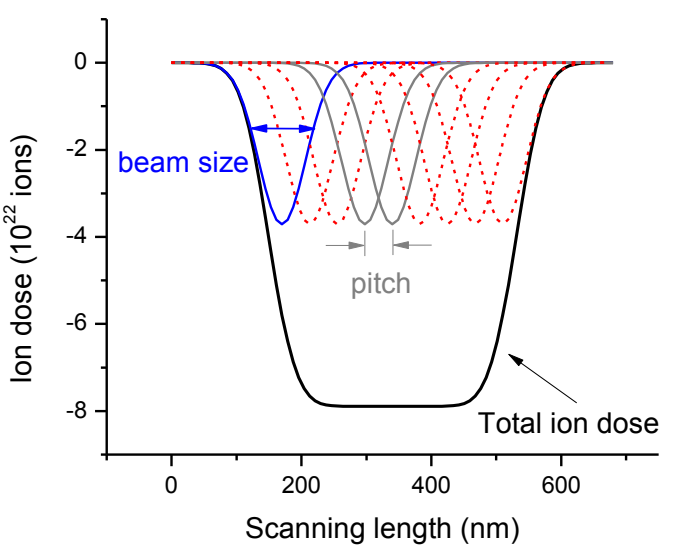

(b)

Figure 6 Ion dose distribution in the FIB machining process. (a) 3D ion dose distribution along the beam scanning direction; (b) cross-sectional view of the ion dose distribution.

The total ion dose is not only related to the dwell time and ion current but also to the number of scanning passes. An investigation on the impact from scanning passes was carried out to further understand the relationship between the total ion dose and the structures' dimensions. For each of the ion current $(0.1 \mathrm{nA}, 1 \mathrm{nA}$ and $5 \mathrm{nA})$, the scanning passes was changed from 2,000 to 300,000, while the dwell time was fixed at $1 \mu \mathrm{s}$. The total ion dose was calculated by equation (3). The fabrication results in addition to the characteristic curves under the above three ion currents are shown in Figure 7. For each current, the dimensions of the structures fabricated under different scanning passes corresponds well with the characteristic curve with a relative error less than $10 \%$. Therefore, it can be concluded that for each ion current the bottom width is constant, while the depth and the mouth width of the gratings are only related to the incident ion dose.

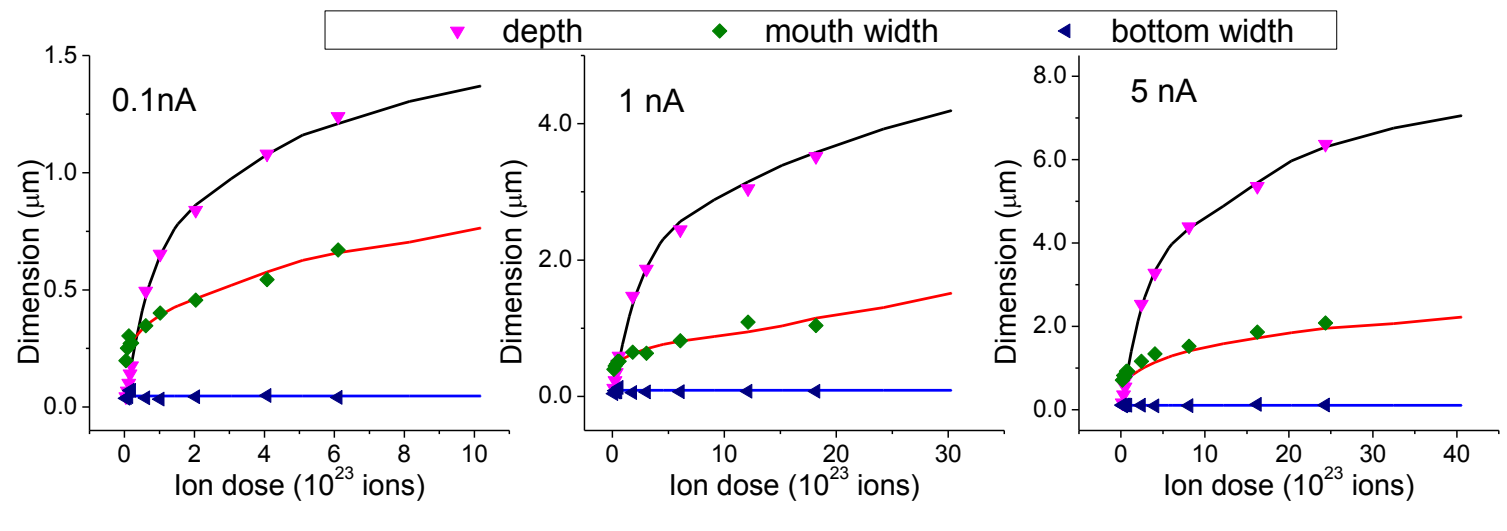

(a)

(b)

(c)

Figure 7 Comparison of the fabrication results under different scanning passes $(2,000 \sim 300,000)$ and the characteristic curves under (a) $0.1 \mathrm{nA}$, (b) $1 \mathrm{nA}$ and (c) $5 \mathrm{nA}$. 
Based on the characteristic curves for each ion current, the cross-sectional profile function $f(x)$ shown in equation (2) can be obtained. As a demonstration of the effectiveness of the proposed method, one nano nano-grating with a depth of $93.2 \mathrm{~nm}$ and one micro-grating with a depth of $3.43 \mu \mathrm{m}$ was chosen as representative. Comparisons of the fitted cross-sectional profile and the experimental results are shown in Figure 8 which reveals that the fitted profile and the experimental results are a very good fit with $\mathrm{R}^{2}=0.998$ for Figure 7 (b) and $\mathrm{R}^{2}=0.993$ for Figure 7 (d).

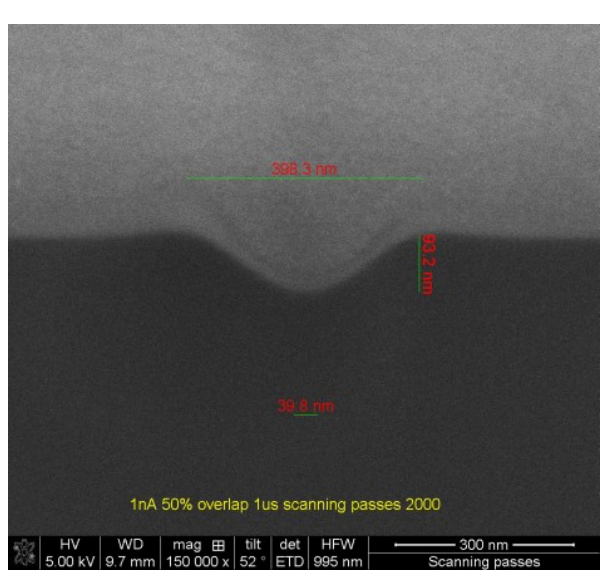

(a)

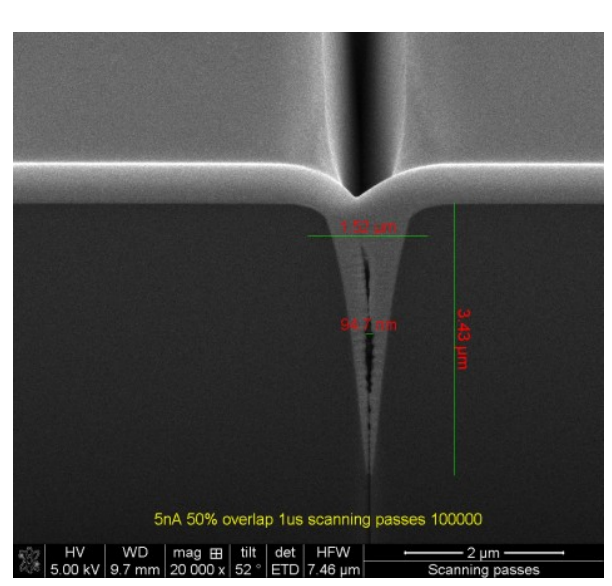

(c)

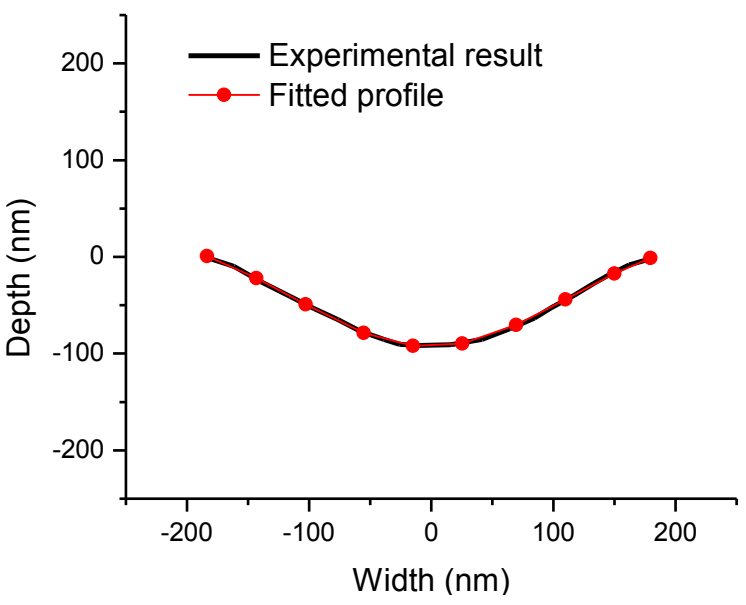

(b)

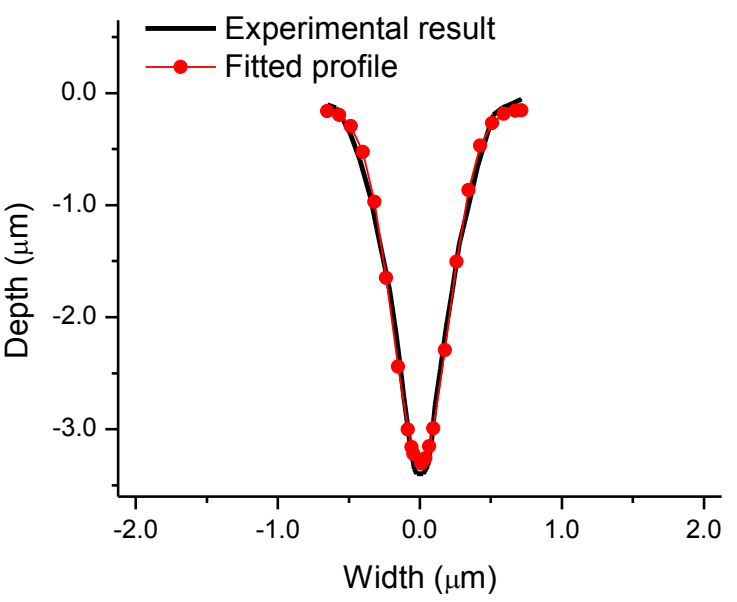

(d)

Figure 8 Comparisons of the fitted cross-sectional profile and the experimental results: (a) and (c) are the SEM images of the cross-sectional profiles of two structures obtained under different fabrication parameters as shown on the images; (b) and (d) show the comparison between the experimental results and the corrected Gaussian fitted profiles.

From the fitted cross-sectional profiles, a database was created for the wide range of FIB machining parameters. The user can select the parameters required for machining with Figure 9 showing the results from haptics simulation of the profiles shown in Figure 8(a) and (c). The user is able to haptically move the substrate into the desired position and then either drill or cut lines across the material using a routing paradigm, simulating the FIB machining process, while 
providing visual and experiential touch feedback. As it can be seen, the haptics simulation provides a viable model and simulation to determine what would be the resulting machined features for a FIB process, which would reduce the time and costs needed for multiple physical machining trials.
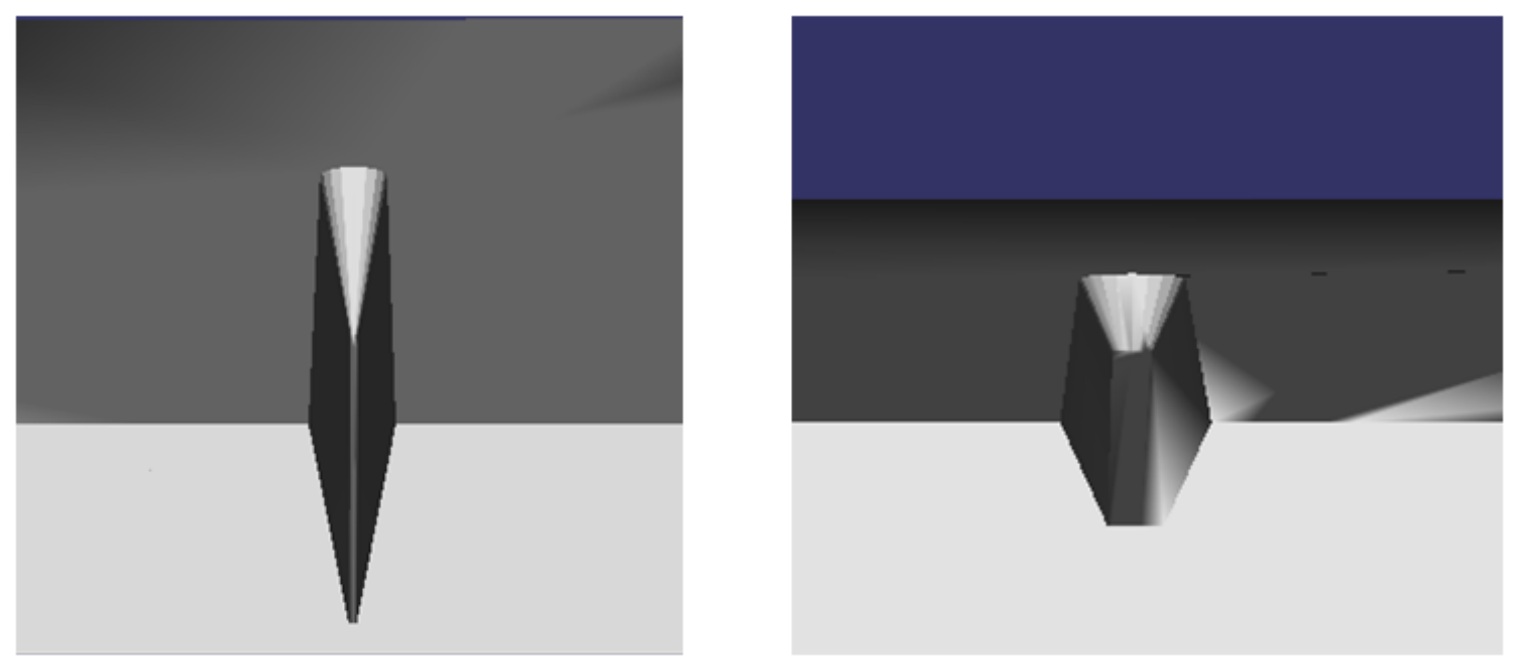

Figure 9 Simulation of FIB machining using a haptics-based program. The cross sectional dimensions are acquired from the experimental data shown in Figure 8.

One application of the above technique is in integrated circuit (IC) editing. As it is shown in Figure 10 (c), two wires underneath the IC package need to be connected for testing purposes. This can be achieved by FIB machining through cutting a small trench between these wires as it is shown in Figure 10 (a) and (b). A layer of platinum was then deposited into the trench as a conductor between the two wires. The width of the trench should be limited below $960 \mathrm{~nm}$ to avoid overlapping with other wires in the circuit, while the depth of the trench should be controlled at $1.7 \mu \mathrm{m}$ in order to reach the wires underneath the package. Before the FIB milling process, the experiment was carried out on a Haptics device to imitate the machining process. According to the simulation results based on haptics device, the right set of fabrication parameters was successfully obtained, based on which, the FIB machining experiment was carried out and the target wires were successfully connected by the platinum bridge as it is shown in Figure 10 (d). 


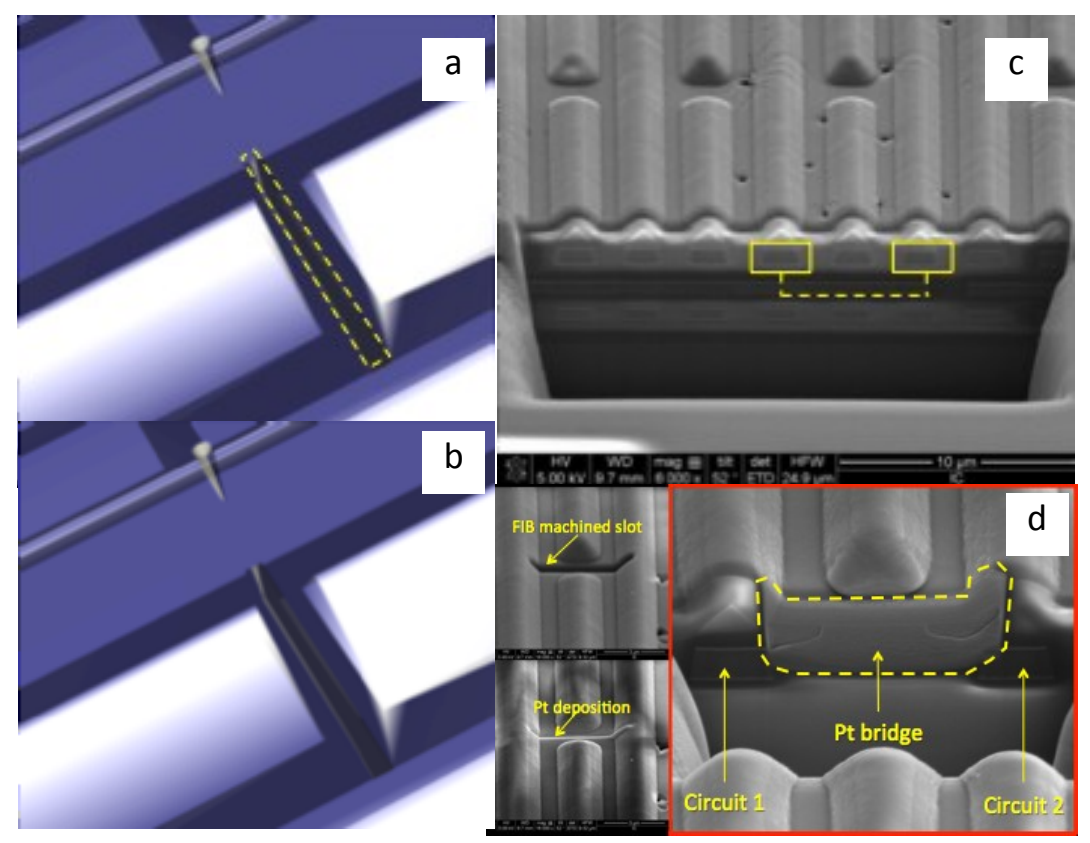

Figure 10 Application of the haptic digital tool in IC editing. (a) (b) Digital planning process; (c) target wires that need to be connected; (d) FIB fabrication results.

\section{CONCLUSIONS}

In this work, a novel haptic design, process planning and manufacturing approach for micro- and nano-fabrication was successfully researched. This provides a true real-time 3D solution for the rapid and accurate manufacture of micro- and nano-gratings using FIB machining. The surface topography simulation reveals that gratings can be characterized by three independent parameters: the mouth width, depth and bottom width. In addition, the cross-sectional profiles of the gratings can be fitted to a corrected Gaussian distribution. The FIB line cutting experiments showed that, for each ion current, the bottom width is constant while the depth and mouth width of the gratings are only related to the incident ion dose. Therefore, the dimensions of the gratings fabricated under each ion current can be described by a specific characteristic curve. For each current, the dimensions of the structures fabricated under the different scanning strategies corresponded closely with the relevant characteristic curve with a relative error of less than $10 \%$. However, the empirical cross-sectional profile dimensions and the experimental results correspond even better - not only the key dimensions fit well with the experimental results but the whole structure profile fits the structure with a $\mathrm{R}^{2}$ of $\sim 0.99$, implying that the empirical modelling of such features and their use in such planning systems may be of benefit in the future.

By introducing the virtual haptics simulation into the FIB machining planning interface, the users can plan and optimize the fabrication parameters for both the design and cost-effective manufacture of micro- and nano-gratings. This approach could potentially provide substantial lead time and cost reductions for what are, traditionally, inherently expensive products. 
In the future, the same principles can also be applied to the planning and manufacture of microand nano-cavities to support the investigation of the machining depth as well as the slope of the side walls, both of which are vital fabrication parameters for the manufacture of hard moulds used for nanoimprint lithography.

\section{FUNDING}

The authors gratefully acknowledge the financial support from EPSRC-funded Heriot-Watt Innovative Manufacturing Research Centre (HW-IMRC) (Project no. 113955).

\section{REFERENCES}

1. Fu Y. Investigation of microlens mold fabricated by focused ion beam technology. Microelectronic engineering. 2001; 56: 333-8.

2. Sun J, Luo X, Ritchie J, Hrncir T. A predictive divergence compensation approach for the fabrication of three-dimensional microstructures using focused ion beam machining. Proceedings of the Institution of Mechanical Engineers, Part B: Journal of Engineering Manufacture. 2012; 226: 229-38.

3. Vasile M, Niu Z, Nassar R, Zhang W, Liu S. Focused ion beam milling: Depth control for threedimensional microfabrication. Journal of Vacuum Science \& Technology B: Microelectronics and Nanometer Structures. 1997; 15: 2350-4.

4. Bryan J. International status of thermal error research (1990). CIRP Annals-Manufacturing Technology. 1990; 39: 645-56.

5. Chen J, Chiou G. Quick testing and modeling of thermally-induced errors of CNC machine tools. International Journal of Machine Tools and Manufacture. 1995; 35: 1063-74.

6. Yang S, Yuan J, Ni J. The improvement of thermal error modeling and compensation on machine tools by CMAC neural network. International Journal of Machine Tools and Manufacture. 1996; 36: 52737.

7. Valladares EG, Castillo, H.I.M., Fletcher, C., Lim, T., Ritchie, J., Yan, X.T., Arnez, V, Hernandez, E. Idealising mesh modelling for haptic enabled services and operands Proceedings of the 37th international Matador conference. London, UK2013, p. 93-6.

8. Lin MC, Otaduy M. Haptic rendering: Foundations, algorithms and applications: AK Peters, Ltd., 2008.

9. Salisbury K, Conti F, Barbagli F. Haptic rendering: introductory concepts. Computer Graphics and Applications, IEEE. 2004; 24: 24-32.

10. Balijepalli A, Kesavadas T. Value-addition of haptics in operator training for complex machining tasks. Journal of Computing and Information Science in Engineering. 2004; 4: 91-7.

11. Lim T, Ritchie J, Corney J, Dewar R, Schmidt K, Bergsteiner K. Assessment of a haptic virtual assembly system that uses physics-based interactions. Assembly and Manufacturing, 2007 ISAM'07 IEEE International Symposium on: IEEE, 2007, p. 147-53.

12. Luo X, Sun J, Ritchie JM, Chang W, Wang W. Deterministic fabrication of nanostructures for plasmonic lens by focused ion beam. The International Journal of Advanced Manufacturing Technology. 2011; 57: 1003-9.

13. Ward J, Utlaut M, Kubena R. Computer simulation of current density profiles in focused ion beams. Journal of Vacuum Science \& Technology B: Microelectronics and Nanometer Structures. 1987; 5: 169-74.

14. OpenSceneGraph: http://www.openscenegraph.org/projects/osg.

15. Open Dynamics Engine: http://www.ode.org/. .

16. osgModeling - A modeling library for OpenSceneGraph: http://code.google.com/p/osgmodeling/.

17. Sensable: http://www.sensable.com/.

18. Wang Z. FIB Nanostructures: Springer, 2013. 